\title{
Application of FDG-PET for detection of malignant lesions in patients with elevated blood tumor markers but without a history of malignancy
}

\author{
HONG-WEI ZHAN ${ }^{1-3}$, WEI XU ${ }^{1,2}$, XIAO-JUAN YE ${ }^{1,2}$, CHUN-LEI ZHAO ${ }^{1-3}$, \\ HONG ZHANG ${ }^{1-3}$, JING LI ${ }^{1,2}$, QIONG YAO ${ }^{1,2}$ and LI-JUN ZHANG ${ }^{1,2}$ \\ ${ }^{1}$ Department of Nuclear Medicine, Second Affiliated Hospital of Zhejiang University College of Medicine; \\ ${ }^{2}$ Zhejiang University Medical PET Center; ${ }^{3}$ Key Laboratory of Medical Molecular Imaging \\ of Zhejiang Province, Hangzhou, Zhejiang, P.R. China
}

Received November 6, 2008; Accepted July 10, 2009

DOI: $10.3892 / \mathrm{mmr} 00000181$

\begin{abstract}
The aim of this study was to evaluate the clinical application of 18-fluorodeoxyglucose positron emission tomography (FDG-PET) imaging for the detection of malignant lesions. A total of 132 patients with increased levels of blood tumor markers but without a prior history of malignancy were examined. The results of FDG-PET and conventional work-up (CWU) including computed tomography (CT), ultrasonography, radionuclide bone scintigraphy and endoscopy were compared. The final diagnosis was based on pathological evidence, other medical imaging results and a follow-up of at least 6 months. There were 61 patients with malignant lesions and 71 without (benign lesions, $n=35$; healthy individuals, $n=36$ ). The average number of elevated tumor markers and the average increase in these tumor markers were greater in the malignant group than in the non-malignant group. FDG-PET imaging revealed that the maximum standardized uptake value (SUVmax) of the major lesion in patients with malignant $(n=61)$ and benign $(n=35)$ tumors was not significantly related to increased levels of tumor markers $(\mathrm{r}=0.10, \mathrm{p}<0.05)$. In patients with malignant lesions and an SUVmax $\geq 3.0$, the diagnostic sensitivity, specificity, accuracy, positive predictive value and negative predictive value of FDG-PET were 95.1, 83.1, 88.6, 82.9 and $95.2 \%$, respectively. CWU identified 61 (100\%) true-positive patients. No statistically significant differences in sensitivity were observed between the results of FDG-PET and CWU ( $>0.05$ ). In 36 healthy subjects without abnormal CWU findings, no abnormal FDG accumulation was revealed by FDG-PET imaging. In conclusion, FDG-PET imaging is a
\end{abstract}

Correspondence to: Dr Hong-Wei Zhan, Department of Nuclear Medicine, Second Affiliated Hospital of Zhejiang University College of Medicine, \#88, Jiefang Road, Hangzhou 310009, Zhejiang, P.R. China E-mail: zhanhw@hotmail.com

Key words: 18-fluorodeoxyglucose, positron emission tomography, tumor markers, cancer detection valuable tool for the detection of malignant lesions in patients with increased levels of blood tumor markers but without a history of malignancy. It is therefore reasonable to apply FDG-PET imaging in situations in which the results of CWU are inconclusive, or when patients wish to limit the number of examinations they must undergo.

\section{Introduction}

The early diagnosis and treatment of malignancies is important for reducing mortality and improving the quality of life of patients. Diagnosis of cancer during its early stages, when it is confined to the organ(s), can greatly increase the chances of complete patient recovery. Blood tumor markers have been used to differentiate cancer patients from healthy individuals or patients with benign conditions $(1,2)$. However, most markers are not specific to a particular tumor, and most cancers have more than one marker associated with their incidence. Additionally, the levels of all tumor markers corresponding to the same tumor may be increased in the serum. Moreover, an increase in tumor markers has been observed in many healthy subjects and in patients with benign lesions such as chronic liver disease, renal failure, tuberculoma and pancreatitis (3-6). Therefore, among individuals with elevated blood tumor markers, it is crucial to differentiate patients with malignant lesions from patients with benign lesions and healthy individuals.

18-Fluorodeoxyglucose positron emission tomography (FDG-PET) is an advanced imaging technique that allows a highly sensitive whole body search for malignant lesions, which are detected by their increased glucose metabolism compared with that of benign tissues. Successful FDG-PET scanning has been performed for a wide variety of cancers (7). Many investigations have shown that FDG-PET is capable of detecting metastases and locally recurrent disease in many types of cancer, including breast, ovarian, colorectal and cervical cancer, using asymptomatically elevated tumor markers. In this regard, it is superior to conventional imaging modalities such as ultrasound, computed tomography (CT), magnetic resonance imaging (MRI), radiography and bone scintigraphy (8-11). However, few studies have evaluated the efficacy of 
FDG-PET for cancer detection in patients with elevated blood tumor markers but without a history of malignancy, and those that have been conducted comprised a small number of patients $(12,13)$. The results are equivocal, and the clinical significance of the application of FDG-PET in such cases is unclear. The purpose of this study was to evaluate the clinical value of FDG-PET imaging for the diagnosis of malignant lesions in patients with increased levels of blood tumor markers but without a history of malignancy.

\section{Materials and methods}

Patients. Patients referred for FDG-PET for the detection of malignant lesions between November 2004 and June 2007 were retrospectively reviewed. All patients had an increase in at least one type of blood tumor marker, but had no history of malignancy. Seven tumor markers were considered, including carcinoembryonic antigen (CEA), carbohydrate antigen 125 (CA125), carbohydrate antigen 153 (CA153), carbohydrate antigen 199 (CA199), carbohydrate antigen 211 (CA211), carbohydrate antigen 242 (CA242) and carbohydrate antigen 724 (CA724). Patients with increased blood $\alpha$-fetoprotein (AFP) or prostate-specific antigen (PSA) levels were excluded, since the detection of hepatocellular and prostate cancer was not a good indicator for FDG-PET imaging (7). Pregnant or lactating patients were also excluded. Patients with a blood glucose reading of $>11 \mathrm{mmol} / \mathrm{l}$ on the day of the study were deemed unsuitable, and another appointment was scheduled. A total of 132 patients was included in this study. FDG-PET imaging was applied to screen for early malignancy in 59 patients, and to differentiate between malignant and benign lesions in 73 patients. The final diagnosis was confirmed by pathological evidence or by a combination of other medical imaging results and a follow-up of at least 6 months.

Conventional work-up (CWU) including ultrasound, CT, MRI, radiography, bone scintigraphy, gastroscopy and colonoscopy was performed using standard procedures to detect the lesions and distant metastases 2 weeks before or 1 week after FDG-PET. The results of these examinations were compared with those of the FDG-PET scans. FDG-PET was performed within 2 weeks of the tumor marker test.

Tumor marker test. Blood samples from all subjects were obtained, and tumor markers were detected. CEA, CA125, CA153, CA199, CA211, CA242 and CA724 were measured by ELISA. The kits for CEA, CA125, CA153, CA199 and CA242 were purchased from CanAg (Gothenburg, Sweden), and the kits for CA211 and CA724 were purchased from LifeKey (USA). The reference range of CEA, CA125, CA153, CA199, CA211, CA242 and CA724 was $<5 \mathrm{ng} / \mathrm{ml}, 35 \mathrm{U} / \mathrm{ml}, 30 \mathrm{U} / \mathrm{ml}$, $37 \mathrm{U} / \mathrm{ml}, 5 \mathrm{ng} / \mathrm{ml}, 20 \mathrm{U} / \mathrm{ml}$ and $18.6 \mathrm{ng} / \mathrm{ml}$, respectively.

Patient preparation and FDG-PET procedure. Patients undergoing FDG-PET imaging fasted overnight, and their glucose level was recorded before the examination. Patients rested in a quiet room for 40-60 min prior to the intravenous injection of $6.66 \mathrm{MBq}(0.18 \mathrm{mCi}) / \mathrm{kg}$ body weight FDG. FDG was produced using a cyclotron (Cypris-HM12S; Sumitomo Heavy Industries, Niihama, Japan) and an automated synthesis system (F100; Sumitomo Heavy Industries).
Table I. Clinical characteristics.

\begin{tabular}{|c|c|}
\hline Characteristic & No. \\
\hline Male/female & $63 / 69$ \\
\hline Age (years) & $59 \pm 15(28-92)$ \\
\hline Malignant lesions $^{\mathrm{a}}$ & 61 \\
\hline Lung cancer & 30 \\
\hline Colorectal cancer & 9 \\
\hline Ovarian cancer & 3 \\
\hline Breast cancer & 3 \\
\hline Gastric cancer & 2 \\
\hline Pancreatic cancer & 4 \\
\hline Thyroid cancer & 1 \\
\hline Unknown origin & 9 \\
\hline Non-malignant lesions ${ }^{\mathrm{a}}$ & 71 \\
\hline Colorectal adenoma or polyps & 11 \\
\hline Tuberculoma & 5 \\
\hline Liver disease & 6 \\
\hline Pancreatitis & 5 \\
\hline Pulmonary inflammatory mass & 3 \\
\hline Pelvic parametritis & 4 \\
\hline Chronic appendicitis & 1 \\
\hline Healthy individuals & 36 \\
\hline
\end{tabular}

${ }^{a}$ No. of evaluable patients, $n=132$.

The whole-body PET scan was carried out $1 \mathrm{~h}$ after the injection of FDG, and the transmission and emission data were obtained using a PET camera (SHR-22000; Hamamatsu Photonics, Hamamatsu, Japan) with a standard BGO crystal that allows for the simultaneous acquisition of 35 image slices with an inter-slice spacing of $3.22 \mathrm{~mm}$. Performance tests of the PET camera showed it to have an intrinsic resolution of 3.78 and $3.63 \mathrm{~mm}$ in the transaxial and axial directions, respectively, and a tomographic sensitivity of $6.44 \mathrm{cps} /(\mathrm{kBq} /$ $\mathrm{ml}$ ) in the $2 \mathrm{D}$ acquisition mode. The axial field of view of the camera was $20 \mathrm{~cm}$. PET scans were performed with the patient in the supine position. Patients were scanned in five bed positions, including the pelvis, abdomen and chest, to the level of the head. A 5-min emission study was performed for each bed position. Immediately after the emission studies, a 3-min transmission scan was performed for each bed position with a rotating $68 \mathrm{Ge} / 68 \mathrm{Ga}$ rod source. Images were acquired in the 2D mode. The PET data were reconstructed using the attenuation-weighted dynamic row-action maximum likelihood algorithm (AW-DRAMA) (14) and re-sliced into transaxial, coronal and sagittal sections with the gray scale. The delayed FDG-PET ( 2 h) images were obtained in cases where abnormal uptake was suspected or if findings were equivocal for the 1-h FDG-PET imaging.

Image interpretation. PET findings for the transaxial, coronal and sagittal sections were analyzed visually. A focal increased uptake of FDG in the surrounding normal tissue in the FDG-PET was interpreted as positive. The maximum standardized uptake 
Table II. Distribution of tumor markers.

\begin{tabular}{lcccc}
\hline Tumor marker & No. of patients & Average concentration (mean \pm SD) & Range & Reference range \\
\hline CEA & 43 & $79.2 \pm 146.8$ & $6-800$ & $<5 \mathrm{ng} / \mathrm{ml}$ \\
CA199 & 68 & $296.3 \pm 383.6$ & $38-1276$ & $<37 \mathrm{U} / \mathrm{ml}$ \\
CA125 & 54 & $234.8 \pm 279.4$ & $36-1121$ & $<35 \mathrm{U} / \mathrm{ml}$ \\
CA153 & 16 & $103.7 \pm 62.5$ & $51-218$ & $<30 \mathrm{U} / \mathrm{ml}$ \\
CA211 & 5 & $65.9 \pm 53.6$ & $6-105$ & $<5 \mathrm{ng} / \mathrm{ml}$ \\
CA242 & 11 & $235.4 \pm 380.8$ & $21.9-1062.7$ & $<20 \mathrm{U} / \mathrm{ml}$ \\
CA724 & 5 & $32.5 \pm 19.9$ & $18.6-63.9$ & $<18.6 \mathrm{ng} / \mathrm{ml}$ \\
\hline
\end{tabular}

Table III. Statistical results for the tumor markers in the different groups.

\begin{tabular}{lccc}
\hline Group & No. $^{\text {a }}$ & No. of elevated markers (mean \pm SD) & Fold increase in markers (mean \pm SD) \\
\hline Non-malignant & & & $5.54 \pm 7.30^{\mathrm{c}}$ \\
Healthy & 36 & $1.19 \pm 0.40^{\mathrm{c}, \mathrm{d}}$ & $6.61 \pm 6.49^{\mathrm{b}}$ \\
Benign & 35 & $1.43 \pm 0.56$ & $6.02 \pm 6.89^{\mathrm{c}}$ \\
Total & 71 & $1.38 \pm 0.51^{\mathrm{b}}$ & $18.07 \pm 26.24$ \\
Malignant & 61 & $1.64 \pm 0.95$ & $11.59 \pm 19.41$ \\
Total & 132 & $1.46 \pm 0.76$ & \\
\hline
\end{tabular}

${ }^{\mathrm{a}}$ No. of patients compared to the malignant group $\left({ }^{\mathrm{b}} \mathrm{p}<0.05,{ }^{\mathrm{c}} \mathrm{p}<0.01\right)$ and compared to the benign group $\left({ }^{\mathrm{d}} \mathrm{p}<0.05,{ }^{\mathrm{e}} \mathrm{p}<0.01\right)$.

value (SUVmax) was then calculated based on the amount of FDG injected, body weight and target tissue uptake in regional attenuation-corrected images for major lesions. Interpretation of the images was conducted independently by two nuclear medicine physicians blinded to the patient data and to the results of other imaging modalities. In cases of disagreement, the final decision was made by consensus. The results of the FDG imaging and the CWU were compared with each other, and then with the final diagnosis based on the histopathological findings or follow-up.

Statistical analysis. For every patient, the number of tumor markers was recorded, and the fold increase in tumor markers compared with the value of the reference range was calculated. The absolute concentration, the types and the fold increase in blood tumor markers are shown as the means \pm SD. Statistical analysis for the comparison of blood tumor marker values in the malignant and non-malignant (benign and healthy) groups was performed using the Student's t-test. Correlation analysis was carried out between the SUVmax of FDG and the fold increase in tumor markers in patients with malignant and benign lesions.

The results of FDG-PET imaging for the diagnosis of patients with malignant lesions were classified into truepositive, true-negative, false-positive or false-negative, with respect to the final diagnosis. From these data, the sensitivity, specificity, accuracy, positive predictive value (PPV) and negative predictive value (NPV) were calculated. Differences between the positive rate from the data obtained by FDG-PET imaging and the positive rate from the CWU were statistically analyzed using the McNemar's test. SPSS 10.0 (Chicago, IL, USA) statistical software was used, and a value of $\mathrm{p}<0.05$ was considered to be statistically significant.

\section{Results}

There were 61 patients with malignant lesions (36 diagnosed by histological findings and 25 by other medical imaging techniques and follow-up) and 71 patients without malignant lesions ( 7 diagnosed by histological findings and 64 by other medical imaging techniques and follow-up; benign lesions, $\mathrm{n}=35$; healthy individuals, $\mathrm{n}=36$ ). CWU was performed before FDG-PET imaging on 109 patients and after FDG-PET imaging on 23 patients. The clinical characteristics of the patients and the final diagnostic results are shown in Table I.

The average number of elevated blood tumor markers was $1.46 \pm 0.76$, and the tumor marker level was on average increased $11.59 \pm 19.41$ times. The average number $(\mathrm{p}<0.05)$ of elevated markers and the average increase $(p<0.01)$ in these markers were higher in the malignant group than in the nonmalignant group. Significant differences between the average number of elevated markers among the three groups were found. The extent of the increase in tumor markers differed significantly between the healthy and malignant groups and the benign and malignant groups, but not between the healthy and benign groups. Detailed results regarding the tumor markers are shown in Tables II and III.

FDG-PET imaging revealed the SUVmax of the major lesion in the malignant $(n=61)$ and benign patients $(n=35)$ to be $5.72 \pm 2.49$ (range 1.50-15.51) and 2.51 \pm 1.56 (range 1.21-8.54), 


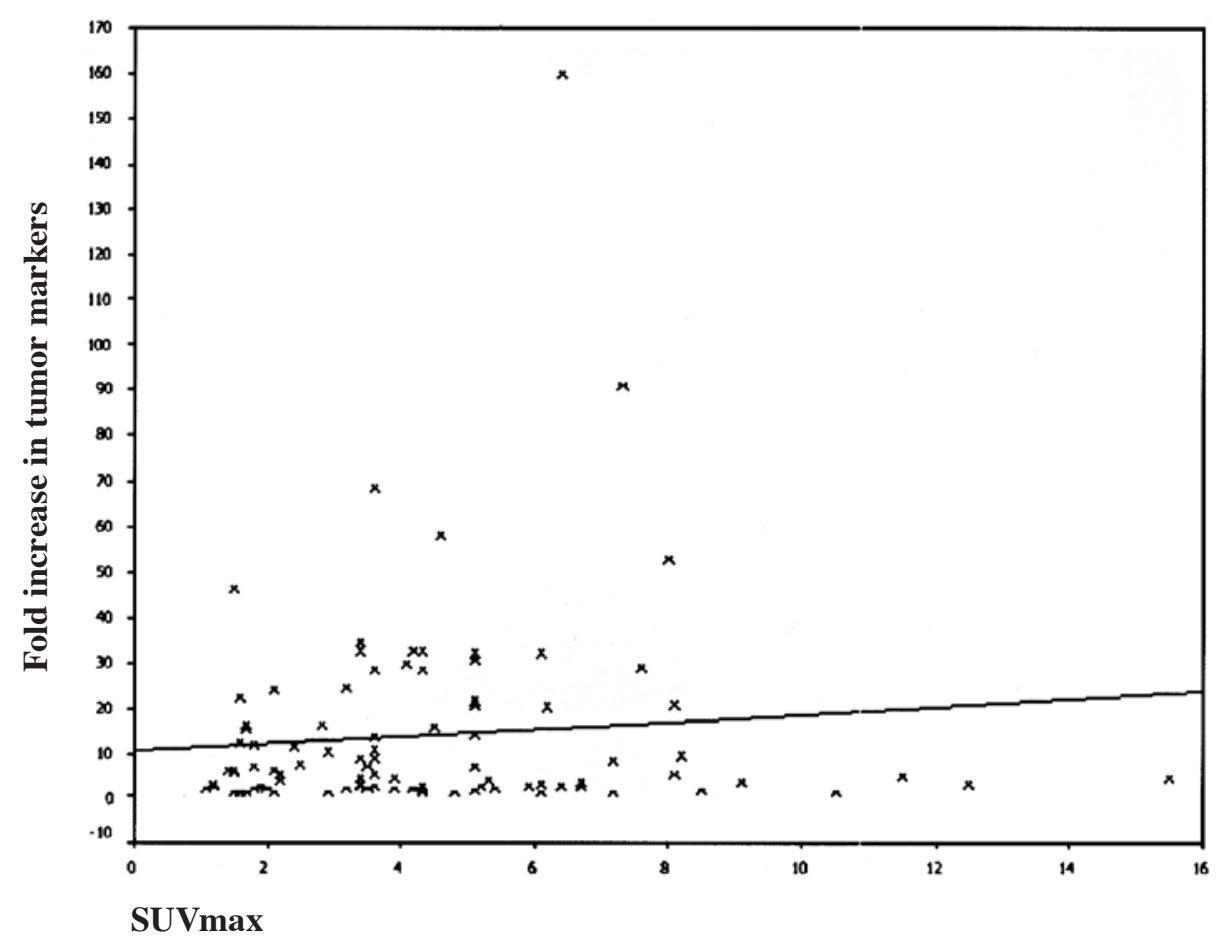

Figure 1. Correlation analysis of the SUVmax of FDG and the extent of increase in tumor markers in patients with malignant and benign lesions ( $\mathrm{r}=0.10$, $\mathrm{p}<0.05$, $\mathrm{n}=96$ ).

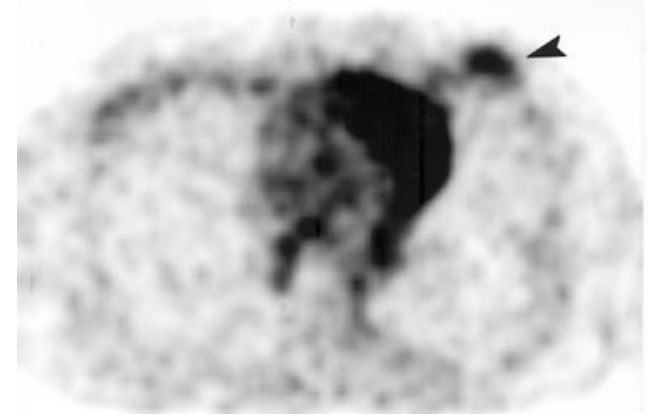

Figure 2. FDG-PET image from a 56-year-old woman with elevated blood CA199 (353 U/ml). FDG accumulation (arrowhead) was noted in the left breast, with an SUVmax of 4.42. No metastatic lesions were found in other sites of the body using FDG-PET. The lesion was histologically confirmed as invasive ductal carcinoma. The mass was $2.2 \mathrm{~cm}$ in diameter.

respectively $(\mathrm{p}<0.01)$. The SUVmax was not significantly related to the extent of the increase in tumor markers (Fig. 1; $\mathrm{r}=0.10, \mathrm{p}<0.05)$. There were 60 patients with a major lesion with an SUVmax $\geq 3.0$ (Fig. 2) and 70 patients with an SUVmax $<3.0$ (Fig. 3), including 36 healthy individuals without focal increased uptake of FDG. When lesions with an SUVmax $\geq 3.0$ were defined as malignant, 58 true-positive and 59 true-negative patients were identified using FDG-PET, while CWU identified 59 true-positive and 57 true-negative patients. The diagnostic sensitivity, specificity, accuracy, PPV and NPV of FDG-PET for malignant patients were 95.1, 83.1, 88.6, 82.9 and $95.2 \%$, respectively. CWU identified 61 (100\%) true-positive patients. No statistically significant differences in sensitivity were observed between FDG-PET and CWU
( $\mathrm{p}>0.05$ ). In 36 healthy subjects without abnormal CWU findings, no abnormal FDG accumulation was revealed by FDG-PET imaging.

Upon FDG-PET imaging, 12 patients were revealed to have a false-positive diagnosis: 3 patients with pulmonary tuberculoma (Fig. 4), 1 with colonic tuberculoma, 3 with pulmonary inflammatory mass, 3 with colorectal adenoma, and 2 with pancreatitis. Three patients had a false-negative diagnosis: 1 with bronchoalveolar carcinoma, 1 with gastric cancer and 1 with colonic cancer.

\section{Discussion}

Elevations in blood tumor markers in serum above the upper limit of the normal range are frequently observed. Marked elevation elicits concern regarding the possibility of occult malignant disease. However, most markers are not specific to a particular tumor. An increase in tumor markers is often observed in healthy subjects and in patients with benign lesions (3-6). Our data produced consistent results: in 132 patients with elevated tumor markers examined by FDG-PET, 61 (46.2\%) were diagnosed with malignant disease and $71(53.8 \%)$ were healthy $(n=36)$ or had benign disease $(n=35)$. Differential diagnosis is therefore vital in individuals with increased blood tumor markers.

The results of this study showed that the number of elevated tumor markers and the extent of the increase were greater in patients with malignant rather than non-malignant disease. However, many patients with malignant disease had just one elevated tumor marker, with a small extent of increase. In contrast, numerous patients with benign disease and healthy individuals had greater increases in a greater number of tumor 

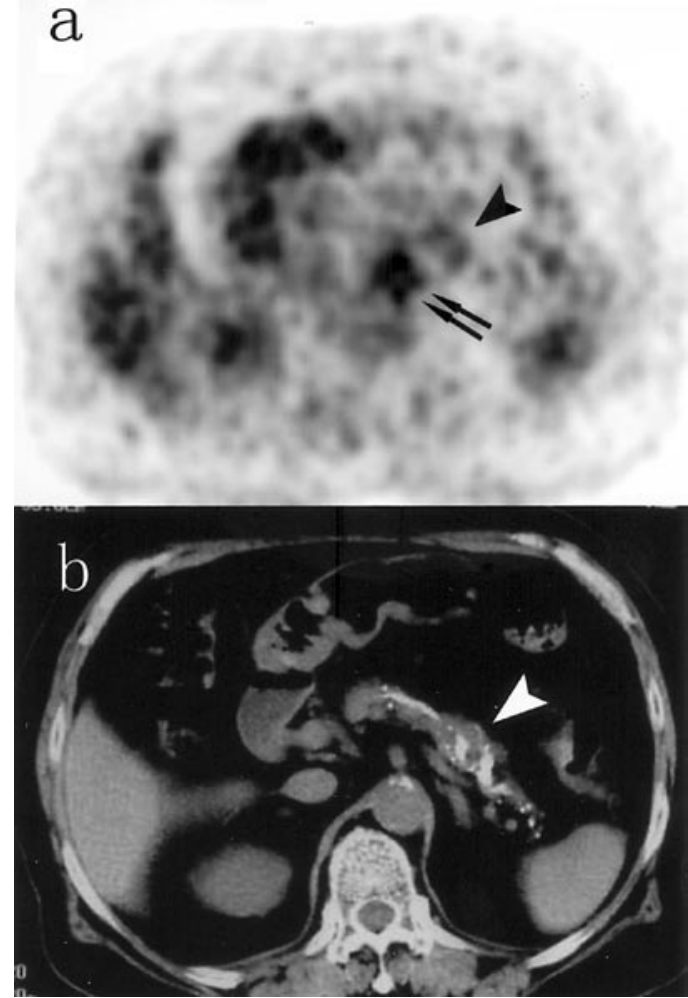

Figure 3. A patient with extremely high blood CA199 (>1200 U/ml) and CA242 (>1062.7 U/ml). (a) FDG-PET imaging showed a low accumulation of FDG in a pancreatic mass (arrowhead, SUVmax: 1.95), and the accumulation of FDG marked by the double arrow was in the abdominal aorta. (b) CT and follow-up confirmed that the patient suffered from chronic calcifying pancreatitis and pancreatic pseudocyst (arrowhead).

markers. The extent of the increase in tumor markers was not correlated with the SUVmax of FDG in the major lesions as determined by FDG-PET imaging. This implies that serum tumor marker levels do not provide sufficient evidence to diagnose malignancy or to predict the tumor glucose metabolic rate and degree of tumor malignancy.

Conventional modalities such as ultrasound, CT, MRI, radiography, gastroscopy and colonoscopy are most commonly used to locate the primary tumor in patients with elevated blood tumor markers (2). CT, MRI and ultrasound have a high resolution and can detect very small lesions. Therefore, they can be used to correctly diagnose many malignant and benign lesions. Despite this, many diagnoses remain indefinite after CT, MRI and ultrasound examination. In such cases, the use of FDG-PET has been considered $(12,13,15-17)$. In our study, on a patient basis, FDG-PET imaging displayed high sensitivity and moderate specificity, and satisfactory accuracy for aiding in clinical diagnosis. The positive rate of FDG-PET was high (95.1\%), with no significant difference compared to that of CWU. This corroborates the findings of Shen et al (12), who demonstrated that whole-body FDG-PET, with the additional evidence of tumor markers, reduces false-negative and falsepositive results obtained by FDG-PET on its own in cancer screening.

There have been few reports concerning the use of FDG-PET imaging in subjects with elevated tumor markers without abnormal findings from conventional imaging. Inoue et al (13) reported that increases in serum CA19-9 are caused
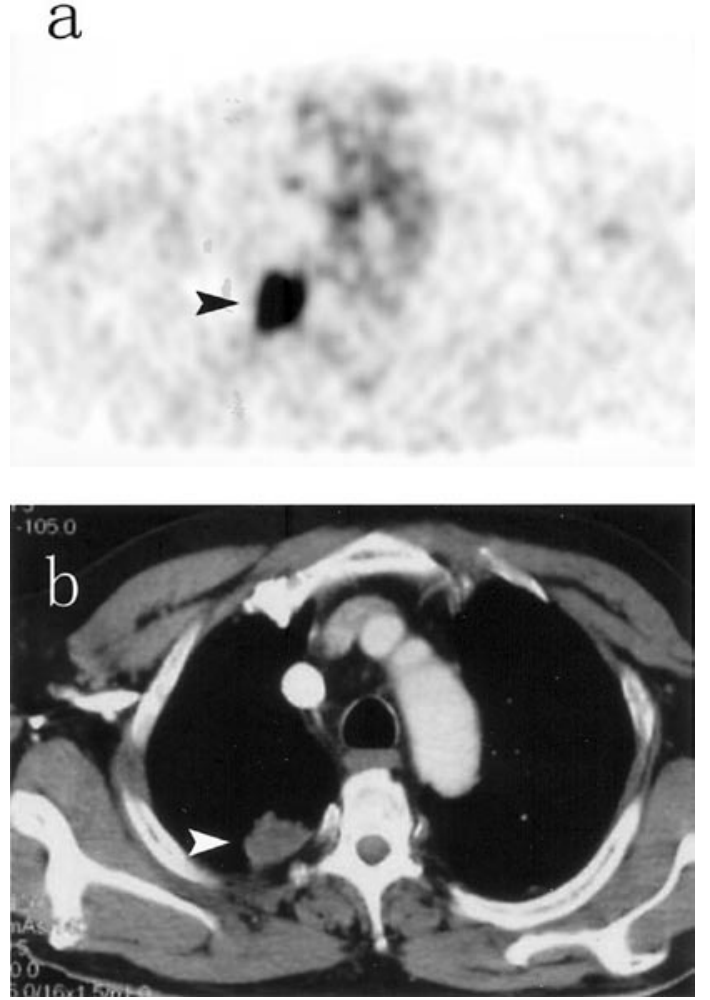

Figure 4. False-positive FDG-PET in a 63-year-old man with pulmonary tuberculosis and elevated blood CA125 (162.3 U/ml). (a) FDG-PET showed a high accumulation of FDG in the right upper lobe (arrowhead, SUVmax: 7.80). (b) CT indicated a mass in the right upper lobe.

by many benign conditions, and that increases in CA19-9 without abnormal findings from conventional imaging do not justify FDG-PET examination, particularly in patients without a history of cancer. Patients with a slight elevation in tumor marker levels and without a history of cancer have a low incidence rate of malignant lesions $(3,18)$. In our study, while 23 patients with benign lesions whose diagnosis was equivocal based on the CWU were correctly diagnosed by FDG-PET, all 36 subjects with abnormal tumor markers levels and without abnormal CWU findings also had no abnormal accumulation of FDG upon PET imaging. Hence, FDG-PET was valuable when applied to patients with inconclusive results after CWU, but its use was not warranted for patients without abnormal CWU findings.

FDG-PET is a non-invasive imaging technique, with the advantage over other imaging methods that all organ systems can be visualized in a single examination (17). This assisted several patients in our study to choose the appropriate CWU modalities according to the FDG-PET results, thus reducing the required number of CWU examinations. FDG-PET imaging may be a promising method for many patients despite its high expense, as it can act as a substitute for other unneeded examinations, some of which may be painful.

FDG-PET has shown high sensitivity in the detection of different types of cancer in many studies (8-11,15-17). A similar high sensitivity was obtained in our study. However, a case of bronchoalveolar carcinoma, one of gastric cancer and one of colonic cancer were not detected by FDG-PET imaging. False-negative FDG-PET results have been reported 
in patients with small lesions (diameter $<1 \mathrm{~cm}$ ) or in those with tumors having a lower degree of FDG accumulation, such as hepatocellular carcinoma, prostate carcinoma, lobular breast carcinoma, renal cell carcinoma, and bronchoalveolar lung carcinoma, as well as in patients with tumors having low FDG uptake related to diffuse, high mucus or muscle uptake, such as gastric carcinoma $(6,7)$. In these cases, other clinical data were required for a correct diagnosis.

There were 12 false-positive results using FDG-PET in our study. They comprised patients with pulmonary tuberculoma $(n=3)$, colonic tuberculoma $(n=1)$, pulmonary inflammatory mass $(n=3)$, colorectal adenoma $(n=3)$ and pancreatitis $(n=2)$. The specificity of FDG-PET for diagnosing malignant lesions has generally been low in previous studies in mainland China (19-22), coincident with the high prevalence of tuberculosis and inflammatory disease (23). Acute or chronic inflammation, abscesses and inflammatory lymphadenopathy and non-specific reactions following radiotherapy may mimic tumor tissue in PET scans (7). This reduces the clinical effectiveness for differentiating benign from malignant lesions in regions with a high prevalence of tuberculosis and inflammatory disease (19). The combination of FDG-PET and other imaging modalities may improve diagnostic accuracy.

This study had the limitations inherent of retrospective analyses. Patient referral bias had an influence on the composition of eligible patients. The final diagnosis of most patients was based on concordant findings with other imaging modalities and follow-up, and only a small number of lesions were verified by histopathology. Further studies are therefore necessary to confirm our findings.

In conclusion, the findings of the present study indicate that FDG-PET imaging is a valuable tool for the detection of malignant lesions in patients with elevated blood tumor markers but without a history of malignancy. However, in patients without abnormal CWU findings, FDG-PET imaging has no additional advantages. These data suggest that FDG-PET imaging may reasonably be applied when the results of CWU are inconclusive, or when patients wish to limit the number of diagnostic examinations they must undergo.

\section{References}

1. Zhang B, Zhang X, Yan H, Xu S, Tang D and Fu W: A novel multi-array immunoassay device for tumor markers based on insert-plug model of piezoelectric immunosensor. Biosens Bioelectro 23: 19-25, 2007.

2. Duffy MJ: Role of tumor markers in patients with solid cancers: A critical review. Eur J Inter Med 18: 175-184, 2007.

3. Roulston JE: Limitations of tumor markers in screening. Br J Surg 77: 961-962, 1990.

4. Maestranzi S, Przemioslo R, Mitchell $\mathrm{H}$ and Sherwood RA: The effect of benign and malignant liver disease on the tumour markers CA19-9 and CEA. Ann Clin Biochem 35: 99-103, 1998.

5. Kanehara H, Bando Y, Tomita M, Kontani M, Takegoshi Y and Tanaka N: Myxedema ascites with an extremely elevated CA125 level: a case report. Endocr J 54: 601-604, 2007.
6. Wang HY and Lin WY: Jejunal tuberculosis: incidental finding on an FDG-PET scan. Kaohsiung J Med Sci 22: 34-38, 2006.

7. Belhocine T, Spaepen K, Dusart M, Castaigne C, Muylie K, Bourgeois P, Bourgeois D, Dierickx L and Flamen P: 18FDG PET in oncology: The best and the worst (Review). Int J Oncol 28: 1249-1261, 2006.

8. Soussan M, Wartski M, Cherel P, Fourme E, Goupil A, Le Stanc E, Callet N, Alexandre J, Pecking AP and Alberini JL: Impact of FDG PET-CT imaging on the decision making in the biologic suspicion of ovarian carcinoma recurrence. Gynecol Oncol 108: 160-165, 2008.

9. Haug AR, Schmidt GP, Klingenstein A, Heinemann V, Stieber P, Priebe M, la Fougère C, Becker C, Hahn K and Tiling R: F-18fluoro-2-deoxyglucose positron emission tomography/computed tomography in the follow-up of breast cancer with elevated levels of tumor markers. J Comput Assist Tomogr 31: 629-634, 2007.

10. Shen YY, Liang JA, Chen YK, Tsai CY and Kao CH: Clinical impact of 18F-FDG-PET in the suspicion of recurrent colorectal cancer based on asymptomatically elevated serum level of carcinoembryonic antigen (CEA) in Taiwan. Hepatogastroenterology 53: 348-350, 2006.

11. Gadducci A, Tana R, Cosio S and Genazzani AR: The serum assay of tumour markers in the prognostic evaluation, treatment monitoring and follow-up of patients with cervical cancer: a review of the literature. Crit Rev Oncol Hematol 66: 10-20, 2008.

12. Shen YY, Su CT, Chen GJ, Chen YK, Liao AC and Tsai FS: The value of $18 \mathrm{~F}$-fluorodeoxyglucose positron emission tomography with the additional help of tumor markers in cancer screening. Neoplasma 50: 217-221, 2003.

13. Inoue K, Okada K, Taki Y, Goto R, Kinomura S, Kaneta $\mathrm{T}$ and Fukuda H: Increase of serum CA19-9 level without an evident lesion on conventional imaging is insufficient for justification of 18FDG-PET examination. Hepatogastroenterology 55: 46-49, 2008.

14. Tanaka E and Kudo H: Subset-dependent relaxation in blockiterative algorithms for image reconstruction in emission tomography. Phys Med Biol 48: 1405-1422, 2003.

15. Zhang L, Jiang X, Li P, Li B and Zhu C: To evaluate retrospectively the impact of 18F-FDG PET on the detection of patients with elevated tumor markers levels. Chin Comput Med Imag 11 (in Chinese): 205-207, 2005.

16. Risum S, Hogdall C, Loft A, et al: The diagnostic value of PET/ CT for primary ovarian cancer - a prospective study. Gynecol Oncol 105: 145-149, 2007.

17. Terauchi T, MuranoT, Daisaki H, Kanou D, Shoda H, Kakinuma R, Hamashima C, Moriyama N and Kakizoe T: Evaluation of wholebody cancer screening using 18F-2-deoxy-2-fluoro-D-glucose positron emission tomography: a preliminary report. Ann Nucl Med 22: 379-385, 2008.

18. Miralles C, Orea M, España P, Provencio M, Sánchez A, Cantos B, Cubedo R, Carcereny E, Bonilla F and Gea T: Cancer antigen 125 associated with multiple benign and malignant pathologies. Ann Surg Oncol 10: 150-154, 2003.

19. Zhan HW, Cheng HF, Ye XJ, Zhao CL, Zhang H, Liu HB, He GQ: Evaluation of lung lesions using FDG gamma-camera PET equipped with a one-inch crystal. Oncol Rep 20: 173-178, 2008.

20. Lan XL, Zhang YX, Wu ZJ, Jia Q, Wei H and Gao ZR: The value of dual time point (18)F-FDG PET imaging for the differentiation between malignant and benign lesions. Clin Radiol 63: 756-764, 2008.

21. Qiu S, Sun H, Yang M, Cong L and Gao J: The role of PET-CT in evaluation of recurrence and metastasis of head-and-neck tumor after definitive treatment (in Chinese). Lin Chung Er Bi Yan Hou Tou Jing Wai Ke Za Zhi 22: 18-21, 2008.

22. Tian J, Yang X, Yu L, Chen P, Xin J, Ma L, Feng H, Tan Y, Zhao Z and $\mathrm{Wu} \mathrm{W}$ : A multicenter clinical trial on the diagnostic value of dual-tracer PET/CT in pulmonary lesions using 3'-deoxy-3'-18Ffluorothymidine and 18F-FDG. J Nucl Med 49: 186-194, 2008.

23. Ministry of Health, PRC: 2007 Chinese Health Statistical Digest. June 1, 2007. Available at: http://www.moh.gov.cn/ newshtml/19165.htm. Accessed Oct. 10, 2007. 